\title{
Introduction to the OQE special issue on Numerical Simulation of Optoelectronic Devices NUSOD'10
}

\author{
Slawomir Sujecki - Mauro Pereira - Enrico Bellotti · \\ Jayanta Sarma · Lu Wei
}

Received: 2 September 2011 / Accepted: 13 October 2011 / Published online: 22 October 2011

(C) Springer Science+Business Media, LLC. 2011

This special issue of Optical and Quantum Electronics contains a selection of papers that were presented at the 10th International Conference on Numerical Simulation of Optoelectronic Devices (NUSOD) in Atlanta, Georgia, USA, September 6-9 2010. NUSOD'10 was chaired by Benjamin Klein and Douglas Yoder from Georgia Institute of Technology, USA, and Joachim Piprek, NUSOD Institute, USA.

The presented papers report on the recent advances in the modelling of semiconductor lasers, semiconductor amplifiers, organic semiconductor devices, light emitting diodes, photo-detectors, integrated optical sensors, integrated optics, and solar cells. The particular topics relate to refinement and optimisation of the devices that are manufactured using well established technological processes and also to the development of novel technologies aimed especially at the opening of new wavelength windows for various applications. The wide scope of the thematic areas that are covered by the papers reflects the diversity of the problems that were discussed at the Conference.

S. Sujecki $(\bowtie)$

Department of Electrical and Electronic Engineering, University of Nottingham,

Nottingham NG7 2RD, UK

e-mail: slawomir.sujecki@nottingham.ac.uk

M. Pereira

Materials and Engineering Research Institute, Hallam University, Sheffield S1 1WB, UK

e-mail: M.Pereira@shu.ac.uk

E. Bellotti

Electrical and Computer Engineering, Boston University, Boston, MA 02215, USA

e-mail: bellotti@bu.edu

J. Sarma

Department of Electronic and Electrical Engineering, University of Bath, Bath BA2 7AY, UK

e-mail: jsarmabath@gmail.com

L. Wei

Shanghai Institute of Technical Physics, Shanghai 200083, China

e-mail: luwei@mail.sitp.ac.cn 
The editors wish to thank all contributing authors for careful preparation of the manuscripts and the reviewers for their work and timely response. We now look forward to NUSOD'11 that will take place in Rome, September 5-8, 2011. 